\title{
A SINGLE - ITEM REPLACEMENT DECISION MODEL FOR REPAIRABLE SPARE PARTS OVER AN INFINITE TIME HORIZON: AN ANALYTICAL SOLUTION
}

\author{
C. O. OGBA and F. E. U. OSAGIEDE
}

(Received 23 March, 2007; Revision Accepted 5 July, 2007)

\begin{abstract}
In this paper, we present an analytical method for determining spare parts replacement over an infinite planning horizon. (The objective is to minimize the total system cost). We develop an exact and simple method for determining the time for equipment replacement or making decision about when to replace equipments, rather than that of continued maintenance of equipments We demonstrate that the optimum replacement time corresponds to when the cost of the next period is greater than the weighted average of expenditures already made. Numerical example is given to illustrate our proposed model
\end{abstract}

Keywords: Replacement, reparable infinte horizon spare parts, interest rate

\subsection{INTRODUCTION}

In wide manufacturing environments product quality and yield is heavily influenced by equipment condition (Sloan 2004) Despite this fact many researchers have either focussed on the maintenance or on the production, omitting the possiblity of actually changing the machine state. As an item of equipment is kept in use for longer period of time it calls for increasing cost of reparrs and maintenance. Hence a time is reached when it becomes more economical to replace the equipment altogether rather than maintaining it.

Literatures on the subject have shown that the problem of maintenance and replacement overlap in practice Graves (1985) model was based on a multi-dimensional Markovian problem. His model gave exact results. However it is more difficult and computationally complex to solve Hau et al. (2007) developed a new approach for forecasting the intermiltent demand of spare parts

Lee (1987). Axsater. (1990), Kukreja et al (2001). Alfredsson and Verrijdt (1999) Yanagi and Sasaki (1992) and Hartanto et al (2005). Grahavac and Chakravarly (2001) gave heuristic methods in solving the replacement problern as a result of the complexity of the problem Sloan and Shanthikumar (2000) "developed a combined production and maintenance scheduling for a multi-product, single-machine production system Terje and Rommert (1997) presented a general framework for optimization of replacement times. Their models included various ages and block replacement models Lee (2005) developed a cost/benefit model for supporting investment strategies about inventory and preventive maintenance in an imperfect production system. Stanislaw (2005) presented a periodic review capacilated lot sizing model with limited backlogging and a possibility of emergency order He considered two re-supply modes; a regular mode and an emergency mode in relation with the system analyzed in this paper, two limitations are encountered in the previous models proposed by other authors, viz. This treatment may be valid for the problems in which the repairable parts are installed on the machines standing on a fixed location, and or the repairable parts are installed on the machine that move from one location to another In this paper the limitations are removed

The primary focus of this paper therefore is to develop an interesting rule for taking optimal equipment replaciement decisions where the equipments are on a fixed location, under the condition that the value of money is time dependent and the equipment is needed for infinite period in the future.

Our main contributions in this paper are; modeling demand, without delays and introducing interest rate. The paper is organised as follows In the next section, we present the basic assumptions and notations used in the model. Section 3 describes the new decision modeling techniques and propositions. In section 4, we present an example of the model application. Section 5 gives the discussion and finally conclusion with directions for further studies in section 6 .

\subsection{NOTATIONS AND ASSUMPTIONS:}

$f(n)$ - Total cost when an item of equipment is replaced after $n$th period.

$r$ - Interest rate.

$c_{n}-$ Initial expenditure on the equipment

$\beta$ - Discount factor

$c, \cdots$ Cost for repairs/maintenance corresponding to the jth period

$n^{*}$ - Optimal replacement time

H - Infinite time horizon

C. O. Ogba, Department of Mathematics, University of Benin, Benın City. Nigeria

F. E. U. Osagiede, Department of Mathematics, University of Benin. Benin City, Nigeria 


\section{Assumptions}

- Charges for maintenance/repairs are paid at the beginning of the corresponding period.

- The equipment is needed over an infinite time horizon $H$

- Money attracts a given interest rate $r$ per period.

- Demand comes from an infinite source. This assumption is reasonable when the expected number of down machines is small relative to the total number of machines

- There exits a series of equal periods numbered $1,2,3$, such that $c_{2}>c_{1}, \quad c_{3}>c_{2}, \ldots c_{n+1}>c_{n}$

- The period $n$ is selected in such a way that it is finite

\subsection{MODEL FORMULATION}

We first note that with interest rate $r$, the present value (PV) of an amount $c$ to be paid $m$ periods in the future is,

$$
p^{\prime}=\frac{c}{(1+m)^{m}}
$$

With the initial expenditure $c_{1}$ and interest rate $r$, we modeled the relationship that the total cost incurred when the equipment is replaced after $n$ period is:

$$
\begin{aligned}
& f(n)=\left[c_{0}+c_{1}+\frac{c_{2}}{(1+r)}+\frac{c_{3}}{(1+r)}+\cdots+\frac{c_{n}}{(1+r)^{n-1}}\right] \\
& +\left[\frac{c_{0}}{(1+r)^{\prime \prime}}+\frac{c_{1}}{(1+r)^{n}}+\frac{c_{2}}{(1+r)^{n+1}}+\cdots+\frac{c_{n}}{(1+r)^{2 n-1}}\right] \\
& +\left[\frac{c_{0}}{(1+r)^{2 n}}+\frac{c_{1}}{(1+r)^{2 n}}+\frac{c_{2}}{(1+r)^{2 n+1}}+\cdots+\frac{c_{n}}{(1+r)^{3 n}}\right]+\cdots
\end{aligned}
$$

This can be simplified as

$$
\begin{aligned}
& f(n)=\left[c_{0}+\sum_{i=1}^{n} \frac{c_{1}}{(1+r)^{\prime-1}}\right]+\frac{1}{(1+r)^{\prime}}\left[c_{0}+\sum_{i=1}^{n} \frac{c_{1}}{(1+r)^{\prime-1}}\right] \frac{1}{(1+r)^{2 n}}\left[c_{0}+\sum_{j=1}^{n} \frac{c_{1}}{(1+r)^{\prime-1}}\right]+\cdots \text { Thus. } \\
& f(n)=\left[c_{0}+\sum_{i=1}^{n} \frac{c_{1}}{(1+r)^{\prime}}\right]\left[1+\frac{1}{(1+r)^{\prime \prime}}+\frac{1}{(1+r)^{2-n}+\cdots}\right]
\end{aligned}
$$

But

$$
\left[1+\left(\frac{1}{1+r}\right)^{n}+\left(\frac{1}{1+r}\right)^{2 n}+\cdots\right] \text { is a geometric series summing to infinity }
$$

Recall that the sum to infinity and $r$ is the common ratio given by

$$
1+\frac{1}{(1+r)^{n}}+\frac{1}{(1+r)^{2 n}}+\ldots \quad\left(\begin{array}{c}
1 \\
(1+r)^{n}
\end{array}\right)^{n} \quad \begin{gathered}
1 \\
1 \\
1(1+r)^{n}
\end{gathered}
$$

As $n$ becomes large, then $\frac{1}{(1+r)^{n}} \rightarrow 0$

$$
f(n)=\frac{\left[c_{0}+\sum_{i=1}^{n}(1+r)^{\prime-1}\right]}{1-(1+r)^{n}}, \text { provided } 1<1
$$

Let $\beta=\frac{1}{1+r}$, equation (2) reduces to

$$
f(n)=\frac{c_{0}+\sum_{i=1}^{n} \beta^{\prime} c^{\prime}}{1 \beta^{\prime \prime}}
$$

The period $n$ is selected in such a way that it is finite 
Equation (3) represents the amount that should be available at the beginning of these periods to replace the equipment every $n$ period (say $n$ years)

The minimum of $f(n)$ which corresponds to the optimum replacement period can be found by tabulating the values of $f(n)$ for various values of $n$

However, we shall demonstrate a rule for obtainıng the minimum of $f(n)$ in the next proposition

\section{Proposition}

Suppose

$$
\begin{aligned}
\therefore>c_{1}, c_{i}>c_{2}, c_{4}>c_{3}, \cdots, c_{n+1}>c_{n}, \text { then the value of } n \text { for which the inequality, } \\
\qquad c_{n+1}>\frac{c_{1}+c_{1}+c_{2} \beta+\cdots+c_{n} \beta^{\prime \prime}}{1+\beta+\beta^{2}+\cdots+\beta^{n-1}} \text {, first holds, corresponds to the optimum replacement }
\end{aligned}
$$

time.

The proposition above leads to the rule: Replace the equipment when the cost of the next period is greater than the weighted mean of all expenditure already made.

\section{Proof:}

The proof of our proposition shall be in two cases

Suppose the minimum of $f(n)$ exists for value of $n^{*}$, then we must have

Case : $\quad f\left(n^{*}+1\right) \cdot f\left(n^{*}\right)>0$

Case II: $\quad f\left(n^{*}-1\right) \quad f\left(n^{*}\right)>0$

Case I: $\quad f\left(n^{*}+1\right) \quad t\left(n^{*}\right)=0$

If we replace $n$ by $(n+1)$ in equation (3). we have

$$
\begin{aligned}
& f(n+1) \quad c_{n}+\sum_{1}^{n+1} f^{\prime}{ }^{\prime} \\
& c_{11}+\sum^{\prime \prime} \beta^{\prime} c^{\prime},+\beta^{\prime \prime} c_{n+3} \\
& 1 \cdot 3^{\prime \prime+1}
\end{aligned}
$$

But from (3).

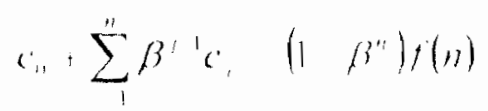

Substitute (5) in (4)

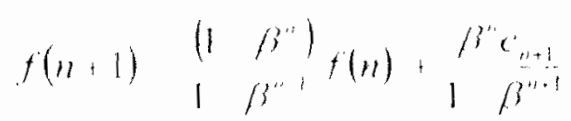

Thus

From which we have

$$
\begin{aligned}
& f(n+1) f(n) \quad \frac{\left(1 \beta^{\prime \prime}\right)}{1} \beta^{n+1} f(n)+\frac{\beta^{\prime \prime} c_{n+1}}{1 \beta^{n+1}} \cdot f(n) \\
& =\left[\frac{1-\beta^{n}-1+\beta^{n+1}}{1 \cdot \beta^{n+1}}\right] f(n)+\frac{\beta^{\prime \prime} c_{n+1}}{1 \beta^{n+1}}
\end{aligned}
$$

$$
f(n+1)-f(n)=\frac{\left[\beta^{n+1}-\beta^{n}\right\rfloor f(n)+\beta^{n} c_{n+1}}{1}
$$

If case i holds, then,

$$
\begin{aligned}
& {\left[\beta^{n+1} \beta^{n}\right] f\left(n^{*}\right)+\beta^{\prime \prime} c_{n+1}>0} \\
& 1 \beta^{\cdots+1}
\end{aligned}
$$


where $1-\beta^{n+1}>0$.

Divide (8) by $\beta^{\prime \prime *}$ we have

$$
\frac{(\beta-1) f\left(n^{*}\right)+c_{n+1}}{1-\beta^{n^{*}+1}}>0
$$

Since $\beta=\frac{1}{1+r}$, and for $r>0$, then $\beta^{\prime \prime+1}=\left(\frac{1}{1+r}\right)^{n+1}<1$

We can write,

which follows that:

$$
\begin{aligned}
& f\left(n^{*}\right)(\beta-1)+c_{n+1}>0 \\
& \left.c_{n+1}>-(1-\beta) f\left(n^{*}\right)\right]
\end{aligned}
$$

$$
\frac{c_{n+1}}{1-\beta}>f\left(n^{*}\right)
$$

Thus

$$
f\left(n^{*}+1\right)-f\left(n^{*}\right)>0 \text { is equivalent to } c_{n}^{*} n_{1}>f\left(n^{*}\right)
$$

Case II when $f\left(n^{*}-1\right)-f\left(n^{*}\right)>0$

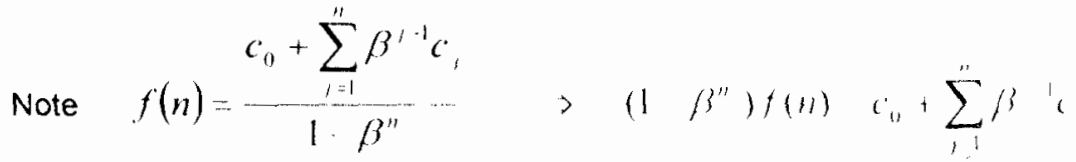

Replace $n$ by $(n-1)$ in equation (3), we have

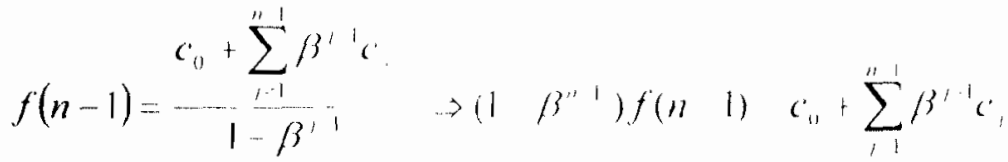

$$
\begin{aligned}
& f(n-1) \frac{c_{0}+\sum_{1=1}^{n} \beta^{\prime} c^{\prime} c_{1}-\beta^{n-1} c_{n}}{1 \beta^{n}}=\frac{c_{0}+\sum_{1=1}^{n} \beta^{\prime} c_{1}}{1-\beta^{n 1}}-\frac{\beta^{n-1} c_{n}}{1-\beta^{n-1}} \\
& =\frac{1}{i} \beta^{\prime \prime} \quad \frac{\beta^{\prime \prime} c_{n}}{1-\beta^{\prime \prime-1}}
\end{aligned}
$$

Thus,

$$
f(n-1)-f(n)=\frac{\left(1-\beta^{n}\right)}{\left(1-\beta^{n-1}\right)}-f(n)-\frac{\beta^{n-1} c_{n}}{1 \cdot \beta^{n-1}}
$$

Since $\beta \quad 1$, then $0<\beta<1$, for $r>0$

From (10)

$$
\begin{aligned}
& c_{0}+\sum_{1=1}^{n} \beta^{\prime-1} c_{,}-\left(1-\beta^{n 1}\right) f(n+1)+\beta^{n 1} c_{n} \\
& =\frac{\left(1-\beta^{\prime \prime}-1+\beta^{\prime \prime}\right) f(n) \quad \beta^{\prime \prime} c^{\prime}}{\left(1-\beta^{\prime \prime} 1\right)}
\end{aligned}
$$

If the inequality in case II holds, then.

$$
\left.=\frac{\left(\beta^{\prime \prime}+\beta^{n}\right) f(n)}{\left(1-\beta^{n}\right)}-\frac{\beta^{\prime \prime} c^{\prime} c_{n}>0}{1} \beta^{\prime \prime}\right)>0
$$

Dividing through (14) by $\beta^{\prime \prime} 1$, we have

$$
\frac{(1-\beta) \cdot f(n)}{1-\beta^{n-1}}-c_{n}>0
$$


Since, $1-\beta^{n}>0$, (15) becomes

$$
(1-\beta) f(n-1)-c_{n}>0
$$

This is equivalent to,

$$
-c_{n}>-(1-\beta) f\left(n^{*}-1\right)
$$

which implies that

$$
\frac{c_{n}}{1-\beta}<f\left(n^{*}-1\right)
$$

Thus

$$
\begin{gathered}
f\left(n^{*}-1\right)-f\left(n^{*}\right)>0 \text { is equivalent to } \\
\frac{c_{n}}{1-\beta}<f\left(n^{*}-1\right)
\end{gathered}
$$

Now, going back to the expression for $f(n)$ in equation (9), we obtain.

$$
c_{n+1}>\frac{(1-\beta)\left(c_{0}+c_{1}+\beta c_{2}+\beta^{2} c_{3}+\cdots+\beta^{n-1} c_{n}\right)}{1-\beta^{n}}
$$

But

$$
\frac{\left(1-\beta^{\prime \prime}\right)}{1-\beta}=1+\beta+\dot{\beta}^{2}+\cdots+\beta^{n-1}
$$

And so equation (12) becomes

$$
c_{n^{+}+1}>\frac{c_{0}+c_{1}+\beta c_{2}+\beta^{2} c_{3}+\cdots+\beta^{n-1} c_{n}}{1+\beta+\beta^{2}+\cdots+\beta^{n-1}}
$$

In a similar manner, substituting the expression for $f(n)$ in equation (16) and replacing $n$ with $n-1$, we have,

$$
c_{n^{*}}<\frac{(1-\beta)}{\left(1-\beta^{n^{\prime}-1}\right)}\left(c_{0}+c_{1}+\beta c_{2}+\beta^{2} c_{3}+\cdots+\beta^{n-2} c_{n-1}\right)
$$

But, $\quad \frac{\left(1-\beta^{n-1}\right)}{1-\beta}=1+\beta+\beta^{2}+\cdots+\beta^{n-2}$

So equation (19) becomes

$$
c_{n}<\frac{c_{0}+c_{1}+\beta c_{2}+\beta^{2} c_{3}+\cdots+\beta^{n-2} c_{\prime \prime-1}}{1+\beta+\beta^{2}+\cdots+\beta^{n-2}}
$$

The RHS of equations (18) and (20) are actually the weighted means of all the costs from period $j=1(1) n$ for (18) and from $j=1(1)(n-1)$ for $(20)$. The weights are the discount factors $1, \beta, \beta^{2}, \cdots$ corresponding to each period. Herice we arrive at the following rule: Replace the equipment when

$$
c_{n^{\prime}+1}>\frac{c_{0}+c_{1}+\beta c_{2}+\cdots+\beta^{\prime \prime-1}}{1+\beta+\beta^{2}+\cdots+\beta^{n-1}}
$$

where the value of $n$ for which the above inequality holds is the optimum replacement time This is now our new equation for optimal replacement time for equipments as at when due.

\subsection{NUMERICAL ILLUSTRATION}

In this section, we illustrate the use of the model by a practical example (Arnold and Kaufmann (1963). Let the interest rate over time be $r=6 \%$, and other parameters are given in the Table 1 below. Table 2 shows the numerical computations.

Table 1: Parameters for the problem with interest rate $6 \%$.

\begin{tabular}{|c|c|c|c|c|c|c|c|c|c|c|c|c|c|}
\hline Year & 0 & 1 & 2 & 3 & 4 & 5 & 6 & 7 & 8 & 9 & 10 & 11 & 12 \\
\hline $\begin{array}{c}c,(\mathrm{~A}) \\
\text { Thousand }\end{array}$ & $1,000,000$ & 50 & 60 & 70 & 90 & 120 & 150 & 180 & 210 & 240 & 300 & 400 & 500 \\
\hline
\end{tabular}

The problem is: When do we replace the existing item? 
Table 2: The Numerical computations and solution.

\begin{tabular}{|c|c|c|c|c|c|c|c|}
\hline Year & $\begin{array}{c}c, \\
\text { thousand }\end{array}$ & $\beta^{j-1}$ & $c_{j} \beta^{\prime-1}$ & $c_{0}+\sum_{j=1}^{n} c_{j} \beta^{\prime-1}$ & $\sum_{j=1}^{n} \beta^{\prime-1}$ & $f(n)$ & $c_{0}+\sum^{n} c_{j} \beta^{\prime-1}$ \\
\hline 1 & 50 & 1.000 & 50.0 & 1,050 & 1.000 & $18,421^{*}$ & $\sum_{j=1}^{n} \beta^{\prime-1}$ \\
\hline 2 & 60 & 0.943 & 56.6 & 1,107 & 1.943 & 10,064 & 570.0 \\
\hline 3 & 70 & 0.890 & 62.3 & 1.169 & 2.833 & 7,306 & 413.0 \\
\hline 4 & 90 & 0.840 & 75.6 & 1,245 & 3.673 & 5,986 & 339.0 \\
\hline 5 & 120 & 0.792 & 95.0 & 1,340 & 4.465 & 5.296 & 300.0 \\
\hline 6 & 150 & 0.747 & 112.1 & 1,452 & 5.212 & 4.922 & 279.0 \\
\hline 7 & 180 & 0.705 & 126.9 & 1,579 & 5.917 & 4,713 & 267.0 \\
\hline 8 & 210 & 0.665 & 139.7 & 1,718 & 6.582 & 4,606 & 261.0 \\
\hline $9^{*}$ & $240^{*}$ & $0.627^{*}$ & $150.5^{*}$ & $1,869^{*}$ & $7.209^{*}$ & $4,581^{*}$ & $259.0^{*}$ \\
\hline 10 & 300 & 0.592 & 177.6 & 2,046 & 7.801 & 4.629 & 262.0 \\
\hline 11 & 400 & 0.558 & 223.2 & 2,270 & 8.359 & 4.799 & 272.0 \\
\hline 12 & 500 & 0.526 & 263.0 & 2,533 & 8.886 & 5.026 & 285.0 \\
\hline
\end{tabular}

For clarity purpose of how the values in Table 2 were obtained, we shall be inquisitive to quickly show how in the first year the values were gotten.

Year 1: [Note $c_{0}=1000$ in thousand]

$$
\begin{aligned}
& c_{1}=c_{1}=50 \\
& \beta^{\prime-1}=\beta^{!-1}=\beta^{0}=0.943^{0}=1 \\
& c, \beta^{\prime-1}=c_{1} \beta^{0}=50 \times 1=50.0 \\
& c_{0}+\sum_{j=1}^{n} c_{i} \beta^{j-1}=c_{1}+\sum_{j=1}^{1} c_{1} \beta^{\prime-1}=1000+50=1.050 \\
& \sum_{i=1}^{n} \beta^{\prime-1}=\sum_{i=1}^{1} \beta^{\prime-1}=\beta^{0}=1.000 \\
& f(n)=\frac{c_{0}+\sum_{i=1}^{\prime \prime} c_{1} \beta^{\prime-1}}{1-\beta^{\prime \prime}}=\frac{1.050}{1-\beta^{\prime}}=\frac{1.050}{1-(0.943)^{\prime}}=\frac{1.050}{1-0.943} \\
& f(n)=18,421=f(1) \\
& \frac{c_{o}+\sum_{i=1}^{n} c, \beta^{J-1}}{\sum_{j=1}^{n} \beta^{J-1}}=\frac{1,050}{1.00}=1,050
\end{aligned}
$$

Similarly this applies to other columns in the table II.

\section{$30 \quad$ REMARK}

1 rom Table II, the minimum of $f(n)$ (in column 7) occurs at $n^{\circ}=9$ and the corresponding value of $f(n)$ is $N 4,581$. Observe that from column 8 of Table 11 , the minimum of $f(n)$ is achieved when the cost of the next period, $\left(\left(1^{*}+1=10\right)\right)$ given as $c_{10}$ is greater than the weighted mean of all expenditures already made That is when

$$
c_{11 j}>\frac{c_{0}+\sum_{j=1}^{9} c_{1} \beta^{\gamma-1}}{\sum_{j=1}^{4} \beta^{\prime-1}} \text { that is. } 300>259 .
$$

Hence, since the cost of the $10^{\text {th }}$ year $c_{10}$ is greater than the weighted mean of all expenditures made up to the $9^{\text {th }}$ year, then the optimum replacement time is the 9th year. It follows that computing the values of $f(n)$ for various values of $n$ coincides with the use of our rule. 
Note that the cost of the $11^{\text {th }}$ year is also greater than the weighted mean of all expenditures made up to the $10^{\text {th }}$ year, i.e $\left(c_{11}=400>262\right)$. A similar situation also holds for the $12^{\text {th }}$ year. However, consideration is given to when the situation first occurs. Thus, since for the first time. the cost of the $10^{\text {th }}$ is greater than the weighted mean of all expenditures made up to the $9^{\text {th }}$ year, then we take the $9^{\text {th }}$ year as the optimum replacement time That is $n^{*}=9$

\subsection{CONCLUSION}

We have developed an analytical solution methodology for determıning the replacement time for repairable spare parts of a single-item over an infinite time horizon $\mathrm{H}$. We demonstrated that the proposed model is simple rule for replacement decision. Obviously, the solution from the computation of $f(n)$ for all possible values of $n$, which minimizes the total cost, $f(n)$ is taken as the optimum replacement time.

Hence if all the assumptions we have considered hold true, replacement of a defender (existing equipment) is due when the cost of keeping it for the next period is greater than the weighted mean of all expenditure already made

Moreover, our method is computationally efficient and can be used to analyze variety of problems. Further research is needed for repairable parts on machines that move from place to place and the cost implication suppose the parts are not readily available. We have presented all mathematical demonstrations in such a way that this work can be understood by other researchers in other fields of study

\section{ACKNOWLEDGEMENTS}

The authors would like to thank the anonymous referees for their helpful comments and suggestions, which indeed significantly improved the paper and gave more clarity to this work.

\section{REFERENCES}

Alfredsson P, Varrijdt, J., 1999. Modeling Emergency Supply Flexibility in a two-echelon Inventory System. Management Science 45: 1416-1431.

Axsater, S., 1990. Modeling Emergency Lateral Transshipments in Inventory Systems. Management Science 6: 13291338

Gravaloc J. and Chakravarty., 2001 Sharing and Lateral Transshipment of Inventory in a Supply Chain with Expensive Low-demand Items. Management Science. 47 579-594.

Graves, S. C., 1985. A multi-echelon Inventory Model for a reparable item with one-for-one Replenishment. Management Science 1 1247-1256.

Hartanto W., Dirk C.. Dirk V O., 2005. Stocking Decisions for Repairable Spare Parts Pooling in a Multi-hub System. Journal Production Economics 93-94, 309-317

Hau Z. S., Zhang, B., Yang, J. and Tan D S., 2007 A new approach for forecasting intermittent demand for spare parts inventories in the process industries. Journal of the Operational Research society 58. 52-61

Kaufmann Arnold, 1963. Methods and Models of Operations Research. Prentice-Hall, Inc., Englewood Cliffs, N.J

Kukreja A. Schmidt, C. P and Miller, D. M., 2001 Stocking Decisions for low-Usage Items in a Multi-location Inventory System. Management Science 47: 1371-1383.

Lee, H. L., 1987. A Multi-echelon Inventory Model for Repairable items with Emergency laterai Transshipments. Management Science 3: 1302-1316

Lee HSU-Hua., 2005. A Cost/benefit model for investments in Inventory and Preventive Maintenance in an Imperfect Production System Computer and Industrial Engineering $48 \quad 55-68$

Sloan, T N., 2004. A Periodic Review Production and Maintenance Model with Random Demand equipment, and Binomial Yield Journal of Operational Research Society 55 647-656

Stanislaw Bylka., 2005 Jurnpike Policies foi Perlodic Review Inventory Model with Emergency Order Int. Journal Production Economics 93-94, 357-373

Sloan, T W. and Shanthikumar J G, 2000 Combined Production and Maintenance Sc.neduling for a Multi-product. Single-Machine Production System Product Uperations Management 9.379-399

Terje Aven and Rommert Dekker, 1997 A Useful Framework for Optimal Replacement Model Reliability Engineering and System Safety 58 61-67

Yanagi, S and Sasakı, M. 1992 An Approximatıon Method for the Problem of a Repairable-item Inventory System with lateral Supply I MA Journal of Mathematics Applied in Business and Industry 3: 305-314. 